\title{
New Existence Results for Fractional Integrodifferential Equations with Nonlocal Integral Boundary Conditions
}

\author{
Ahmed Alsaedi, ${ }^{1}$ Sotiris K. Ntouyas, ${ }^{1,2}$ and Bashir Ahmad ${ }^{1}$ \\ ${ }^{1}$ Nonlinear Analysis and Applied Mathematics (NAAM) Research Group, Department of Mathematics, Faculty of Science, \\ King Abdulaziz University, P.O. Box 80203, Jeddah 21589, Saudi Arabia \\ ${ }^{2}$ Department of Mathematics, University of Ioannina, 45110 Ioannina, Greece
}

Correspondence should be addressed to Ahmed Alsaedi; aalsaedi@hotmail.com

Received 15 June 2014; Accepted 14 October 2014

Academic Editor: Erdal Karapinar

Copyright (C) 2015 Ahmed Alsaedi et al. This is an open access article distributed under the Creative Commons Attribution License, which permits unrestricted use, distribution, and reproduction in any medium, provided the original work is properly cited.

\begin{abstract}
We consider a boundary value problem of fractional integrodifferential equations with new nonlocal integral boundary conditions of the form: $x(0)=\beta x(\theta), x(\xi)=\alpha \int_{\eta}^{1} x(s) d s$, and $0<\theta<\xi<\eta<1$. According to these conditions, the value of the unknown function at the left end point $t=0$ is proportional to its value at a nonlocal point $\theta$ while the value at an arbitrary (local) point $\xi$ is proportional to the contribution due to a substrip of arbitrary length $(1-\eta)$. These conditions appear in the mathematical modelling of physical problems when different parts (nonlocal points and substrips of arbitrary length) of the domain are involved in the input data for the process under consideration. We discuss the existence of solutions for the given problem by means of the Sadovski fixed point theorem for condensing maps and a fixed point theorem due to O’Regan. Some illustrative examples are also presented.
\end{abstract}

\section{Introduction}

We consider a boundary value problem of fractional differential equations with nonlocal integral boundary conditions given by

$$
\begin{gathered}
{ }^{c} D^{q} x(t)=A f(t, x(t))+B I^{r} g(t, x(t)), \quad t \in[0,1], \\
x(0)=\beta x(\theta), \quad x(\xi)=\alpha \int_{\eta}^{1} x(s) d s, \\
0<\theta<\xi<\eta<1,
\end{gathered}
$$

where ${ }^{c} D^{q}$ denotes the Caputo fractional derivative of order $q$, $f:[0,1] \times \mathbb{R} \rightarrow \mathbb{R}$ is a given continuous function, $1<q \leq 2$, $0<r<1$, and $\alpha, \beta, A, B$ are real constants.

Here we remark that the boundary conditions introduced in the problem (1) are of nonlocal strip type and describe the situation when the receptors at the end points of the boundary are influenced by the nonlocal contributions due to interior points and strips of the domain for the problem.
For practical examples, see $[1,2]$. The problem (1) can also be termed as a five-point nonlocal fractional boundary value problem.

In recent years, several aspects of fractional boundary value problems, ranging from theoretical analysis to numerical simulation, have been investigated. The nonlocal nature of fractional order differential operators has significantly contributed to the popularity and development of the subject. As a matter of fact, this characteristic of such operators help to understand the memory and hereditary properties of many useful materials and processes. For details and applications of fractional differential equations in physical and technical sciences such as biology, physics, biophysics, chemistry, statistics, economics, blood flow phenomena, control theory, and signal and image processing, see [3-6]. For some recent works on nonlocal fractional boundary value problems, we refer the reader to the papers [7-11], while the results based on monotone method for such problems can be found in $[12,13]$. In [14], the limit properties of positive solutions of fractional boundary value problems have been discussed. 
Fractional differential inclusions supplemented with different kinds of boundary conditions have also been studied by several researchers, for instance, see [15-20].

The paper is organized as follows. In Section 2, we recall some basic definitions from fractional calculus and establish an auxiliary lemma which plays a pivotal role in the sequel. Section 3.1 contains an existence result for the problem (1) which is established by applying Sadovskii's fixed point theorem for condensing maps. In Section 3.2, we show the existence of solutions for the problem (1) by means of a fixed point theorem due to O'Regan.

\section{Preliminaries}

In this section, some basic definitions on fractional calculus and an auxiliary lemma are presented $[3,4]$.

Definition 1. The Riemann-Liouville fractional integral of order $q$ for a continuous function $g$ is defined as

$$
I^{q} g(t)=\frac{1}{\Gamma(q)} \int_{0}^{t} \frac{g(s)}{(t-s)^{1-q}} d s, \quad q>0,
$$

provided the integral exists.

Definition 2. For at least $n$-times continuously differentiable function $g:[0, \infty) \rightarrow \mathbb{R}$, the Caputo derivative of fractional order $q$ is defined as

$$
\begin{array}{r}
{ }^{c} D^{q} g(t)=\frac{1}{\Gamma(n-q)} \int_{0}^{t}(t-s)^{n-q-1} g^{(n)}(s) d s, \\
n-1<q<n, \quad n=[q]+1,
\end{array}
$$

where $[q]$ denotes the integer part of the real number $q$.

Lemma 3. For any $y \in C([0,1], \mathbb{R})$ the unique solution of the linear fractional boundary value problem

$$
\begin{gathered}
{ }^{c} D^{q} x(t)=y(t), \quad t \in[0,1], 1<q \leq 2, \\
x(0)=\beta x(\theta), \quad x(\xi)=\alpha \int_{\eta}^{1} x(s) d s, \\
0<\theta<\xi<\eta<1,
\end{gathered}
$$

is

$$
\begin{aligned}
x(t)= & \int_{0}^{t} \frac{(t-s)^{q-1}}{\Gamma(q)} y(s) d s \\
& +\frac{\beta\left(\xi-(\alpha / 2)\left(1-\eta^{2}\right)\right)}{Q} \int_{0}^{\theta} \frac{(\theta-s)^{q-1}}{\Gamma(q)} y(s) d s \\
& +\frac{\alpha \beta \theta}{Q}\left(\int_{0}^{1} \frac{(1-s)^{q}}{\Gamma(q+1)} y(s) d s-\int_{0}^{\eta} \frac{(\eta-s)^{q}}{\Gamma(q+1)} y(s) d s\right) \\
& -\frac{\beta \theta}{Q} \int_{0}^{\xi} \frac{(\xi-s)^{q}}{\Gamma(q)} y(s) d s
\end{aligned}
$$

$$
\begin{aligned}
& +\frac{t}{Q}\left[\alpha ( 1 - \beta ) \left(\int_{0}^{1} \frac{(1-s)^{q}}{\Gamma(q+1)} y(s) d s\right.\right. \\
& \left.\quad-\int_{0}^{\eta} \frac{(\eta-s)^{q}}{\Gamma(q+1)} y(s) d s\right) \\
& \quad-(1-\beta) \int_{0}^{\xi} \frac{(\xi-s)^{q}}{\Gamma(q)} y(s) d s \\
& \left.\quad-\beta(1-\alpha(1-\eta)) \int_{0}^{\theta} \frac{(\theta-s)^{q-1}}{\Gamma(q)} y(s) d s\right],
\end{aligned}
$$

where

$$
Q=(1-\beta)\left(\xi-\frac{\alpha}{2}\left(1-\eta^{2}\right)\right)+\beta \theta(1-\alpha(1-\eta)) \neq 0 .
$$

Proof. It is well known that the general solution of the fractional differential equation in (4) can be written as

$$
x(t)=c_{0}+c_{1} t+\int_{0}^{t} \frac{(t-s)^{q-1}}{\Gamma(q)} y(s) d s
$$

where $c_{0}, c_{1} \in \mathbb{R}$ are arbitrary constants.

Applying the given boundary conditions, we obtain the following system

$$
\begin{gathered}
(1-\beta) c_{0}-\beta \theta c_{1}=\beta \int_{0}^{\theta} \frac{(\theta-s)^{q-1}}{\Gamma(q)} y(s) d s \\
(1-\alpha(1-\eta)) c_{0}+\left(\xi-\frac{\alpha}{2}\left(1-\eta^{2}\right)\right) c_{1} \\
=\alpha\left(\int_{0}^{1} \frac{(1-s)^{q}}{\Gamma(q+1)} y(s) d s-\int_{0}^{\eta} \frac{(\eta-s)^{q}}{\Gamma(q+1)} y(s) d s\right) \\
\quad-\int_{0}^{\xi} \frac{(\xi-s)^{q}}{\Gamma(q)} y(s) d s,
\end{gathered}
$$

from which we get

$$
\begin{aligned}
& c_{0}= \frac{\beta\left(\xi-(\alpha / 2)\left(1-\eta^{2}\right)\right)}{Q} \int_{0}^{\theta} \frac{(\theta-s)^{q-1}}{\Gamma(q)} y(s) d s \\
&+\frac{\alpha \beta \theta}{Q}\left(\int_{0}^{1} \frac{(1-s)^{q}}{\Gamma(q+1)} y(s) d s-\int_{0}^{\eta} \frac{(\eta-s)^{q}}{\Gamma(q+1)} y(s) d s\right) \\
&-\frac{\beta \theta}{Q} \int_{0}^{\xi} \frac{(\xi-s)^{q}}{\Gamma(q)} y(s) d s, \\
& c_{1}= \frac{1}{Q}\left[\alpha ( 1 - \beta ) \left(\int_{0}^{1} \frac{(1-s)^{q}}{\Gamma(q+1)} y(s) d s\right.\right. \\
&\left.-\int_{0}^{\eta} \frac{(\eta-s)^{q}}{\Gamma(q+1)} y(s) d s\right)
\end{aligned}
$$




$$
\begin{aligned}
& -(1-\beta) \int_{0}^{\xi} \frac{(\xi-s)^{q}}{\Gamma(q)} y(s) d s \\
& \left.-\beta(1-\alpha(1-\eta)) \int_{0}^{\theta} \frac{(\theta-s)^{q-1}}{\Gamma(q)} y(s) d s\right] .
\end{aligned}
$$

Substituting the values of $c_{0}, c_{1}$ in (7), we get (5). This completes the proof.

\section{Existence Results}

We denote by $\mathscr{C}=C([0,1], \mathbb{R})$ the Banach space of all continuous functions from $[0,1] \rightarrow \mathbb{R}$ endowed with the norm defined by $\|x\|=\sup \{|x(t)|: t \in[0,1]\}$. Also by $L^{1}([0,1], \mathbb{R})$ we denote the Banach space of measurable functions $x:[0,1] \rightarrow \mathbb{R}$ which are Lebesgue integrable and normed by $\|x\|_{L^{1}}=\int_{0}^{1}|x(t)| d t$.

In the following we will give two existence results for the problem (1), one with the help of Sadovskii's fixed point theorem and the other based on a fixed point theorem due to O'Regan in [21].

\subsection{Existence Results via Sadovskii’s Fixed Point Theorem}

Definition 4. Let $M$ be a bounded set in metric space $(X, d)$; then Kuratowskii measure of noncompactness, $\alpha(M)$, is defined as $\inf \{\epsilon: M$ covered by finitely many sets such that the diameter of each set $\leq \epsilon\}$.

Definition 5 (see [22]). Let $\Phi: D(\Phi) \subseteq X \rightarrow X$ be a bounded and continuous operator on Banach space $X$. Then $\Phi$ is called a condensing map if $\alpha(\Phi(B))<\alpha(B)$ for all bounded sets $B \subset D(\Phi)$, where $\alpha$ denotes the Kuratowski measure of noncompactness.

Lemma 6 (see [23, Example 11.7]). The map $K+C$ is a $k$-set contraction with $0 \leq k<1$ and is thus condensing, if

(i) $K, C: D \subseteq X \rightarrow X$ are operators on the Banach space $X$;

(ii) $K$ is $k$-contractive; that is,

$$
\|K x-K y\| \leq k\|x-y\|
$$

for all $x, y \in D$ and fixed $k \in[0,1)$;

(iii) $C$ is compact.

Theorem 7 (see [24]). Let B be a convex, bounded, and closed subset of a Banach space $X$ and let $\Phi: B \rightarrow B$ be a condensing map. Then $\Phi$ has a fixed point. by

In view of Lemma 3, we define an operator $\mathscr{P}: \mathscr{C} \rightarrow \mathscr{C}$

$$
(\mathscr{P} x)(t)=\left(\mathscr{P}_{1} x\right)(t)+\left(\mathscr{P}_{2} x\right)(t), \quad t \in[0,1]
$$

where

$$
\begin{aligned}
& \left(\mathscr{P}_{1} x\right)(t) \\
& =A \int_{0}^{t} \frac{(t-s)^{q-1}}{\Gamma(q)} f(s, x(s)) d s \\
& +A \frac{\beta\left(\xi-(\alpha / 2)\left(1-\eta^{2}\right)\right)}{Q} \int_{0}^{\theta} \frac{(\theta-s)^{q-1}}{\Gamma(q)} f(s, x(s)) d s \\
& +A \frac{\alpha \beta \theta}{Q}\left(\int_{0}^{1} \frac{(1-s)^{q}}{\Gamma(q+1)} f(s, x(s)) d s\right. \\
& -A \frac{\beta \theta}{Q} \int_{0}^{\xi} \frac{(\xi-s)^{q}}{\Gamma(q)} f(s, x(s)) d s \\
& +\frac{A t}{Q}\left[\alpha ( 1 - \beta ) \left(\int_{0}^{1} \frac{(1-s)^{q}}{\Gamma(q+1)} f(s, x(s)) d s\right.\right. \\
& -(1-\beta(1-\alpha(s)) d s) \\
& -\int_{0}^{\xi} \frac{(\xi-s)^{q}}{\Gamma(q)} f(s, x(s)) d s \\
& \Gamma(q+1)
\end{aligned}
$$

$$
\begin{aligned}
& \left(\mathscr{P}_{2} x\right)(t) \\
& =B \int_{0}^{t} \frac{(t-s)^{q-1}}{\Gamma(q)} \int_{0}^{s} \frac{(s-u)^{r-1}}{\Gamma(r)} g(u, x(u)) d u d s \\
& +B \frac{\beta\left(\xi-(\alpha / 2)\left(1-\eta^{2}\right)\right)}{Q} \\
& \times \int_{0}^{\theta} \frac{(\theta-s)^{q-1}}{\Gamma(q)} \int_{0}^{s} \frac{(s-u)^{r-1}}{\Gamma(r)} g(u, x(u)) d u d s \\
& +B \frac{\alpha \beta \theta}{Q}\left(\int_{0}^{1} \frac{(1-s)^{q}}{\Gamma(q+1)} \int_{0}^{s} \frac{(s-u)^{r-1}}{\Gamma(r)} g(u, x(u)) d u d s\right. \\
& -B \frac{\beta \theta}{Q} \int_{0}^{\xi} \frac{(\xi-s)^{q}}{\Gamma(q)} \int_{0}^{s} \frac{(s-u)^{r-1}}{\Gamma(r)} g(u, x(u)) d u d s \\
& +\frac{B t}{Q}[\alpha(1-\beta) \\
& \times\left(\int_{0}^{1} \frac{(1-s)^{q}}{\Gamma(q+1)} \int_{0}^{s} \frac{(s-u)^{r-1}}{\Gamma(r)} g(u, x(u)) d u d s\right) \\
& \hline(r) \\
& \hline
\end{aligned}
$$




$$
\begin{gathered}
-\int_{0}^{\eta} \frac{(\eta-s)^{q}}{\Gamma(q+1)} \int_{0}^{s} \frac{(s-u)^{r-1}}{\Gamma(r)} \\
\times g(u, x(u)) d u d s) \\
-(1-\beta) \int_{0}^{\xi} \frac{(\xi-s)^{q}}{\Gamma(q)} \int_{0}^{s} \frac{(s-u)^{r-1}}{\Gamma(r)} \\
-\beta(1-\alpha(1-\eta)) \\
\times g(u, x(u)) d u d s \\
\left.\times \int_{0}^{\theta} \frac{(\theta-s)^{q-1}}{\Gamma(q)} \int_{0}^{s} \frac{(s-u)^{r-1}}{\Gamma(r)} g(u, x(u)) d u d s\right] .
\end{gathered}
$$

Theorem 8. Let $f, g:[0,1] \times \mathbb{R} \rightarrow \mathbb{R}$ be continuous functions satisfying the following conditions.

$\left(H_{1}\right) f$ satisfies the Lipschitz condition:

$$
\begin{array}{r}
|f(t, x)-f(t, y)| \leq L|x-y|, \\
L>0, \quad \forall(t, x),(t, y) \in[0,1] \times \mathbb{R},
\end{array}
$$

$\left(H_{2}\right)$ there exist a function $m \in C\left([0,1], \mathbb{R}^{+}\right)$and a nondecreasing function $\psi: \mathbb{R}^{+} \rightarrow \mathbb{R}^{+}$such that

$$
|g(t, x)| \leq m(t) \psi(\|x\|), \quad \forall(t, x) \in[0,1] \times \mathbb{R} .
$$

Then the boundary value problem (1) has at least one solution provided that

$$
\begin{aligned}
\gamma:=|A| L\{ & \frac{1}{\Gamma(q+1)}+\frac{\left|\beta\left(\xi-(\alpha / 2)\left(1-\eta^{2}\right)\right)\right| \theta^{q}}{|Q| \Gamma(q+1)} \\
& +\frac{|\alpha \beta| \theta}{|Q|}\left(\frac{1}{\Gamma(q+2)}+\frac{\eta^{q+1}}{\Gamma(q+2)}\right) \\
& +\frac{|\beta| \theta \xi^{q}}{|Q| \Gamma(q+1)}+\frac{1}{|Q|} \\
& \times\left[|\alpha(1-\beta)|\left(\frac{1}{\Gamma(q+2)}+\frac{\eta^{q+1}}{\Gamma(q+2)}\right)\right. \\
& \left.\left.+\frac{|1-\beta| \xi^{q}}{\Gamma(q+1)}+\frac{|\beta(1-\alpha(1-\eta))| \theta^{q}}{\Gamma(q+1)}\right]\right\}
\end{aligned}
$$

Proof. Let $B_{v}=\{x \in \mathscr{C}:\|x\| \leq \nu\}$ be a closed bounded and convex subset of $\mathscr{C}:=C([0,1], \mathbb{R})$, where $\nu$ will be fixed later. We define a map $\mathscr{P}: B_{v} \rightarrow \mathscr{C}$ as

$$
(\mathscr{P} x)(t)=\left(\mathscr{P}_{1} x\right)(t)+\left(\mathscr{P}_{2} x\right)(t), \quad t \in[0,1]
$$

where $\mathscr{P}_{1}$ and $\mathscr{P}_{2}$ are defined by (12) and (13), respectively. Notice that the problem (1) is equivalent to a fixed point problem $\mathscr{P}(x)=x$.

Step $1\left((\mathscr{P} x)\left(B_{v}\right) \subset B_{v}\right)$. For that, we set $M=\sup _{t \in[0,1]}|f(t, 0)|$ and select $v \geq \omega /(1-\gamma)$, where

$$
\begin{aligned}
\omega=|A| M[ & \frac{1}{\Gamma(q+1)} \\
& +\frac{\left|\beta\left(\xi-(\alpha / 2)\left(1-\eta^{2}\right)\right)\right| \theta^{q}}{|Q| \Gamma(q+1)} \\
& +\frac{|\alpha \beta| \theta}{|Q|}\left(\frac{1}{\Gamma(q+2)}+\frac{\eta^{q+1}}{\Gamma(q+2)}\right) \\
& +\frac{|\beta| \theta \xi^{q}}{|Q| \Gamma(q+1)}+\frac{1}{|Q|} \\
& \times\left\{|\alpha(1-\beta)|\left(\frac{1}{\Gamma(q+2)}+\frac{\eta^{q+1}}{\Gamma(q+2)}\right)\right. \\
& \left.\left.+\frac{|1-\beta| \xi^{q}}{\Gamma(q+1)}+\frac{|\beta(1-\alpha(1-\eta))| \theta^{q}}{\Gamma(q+1)}\right\}\right]
\end{aligned}
$$

$+|B|\|m\| \psi(r)$

$$
\begin{aligned}
& \times\left[\frac{1}{\Gamma(q+r+1)}+\frac{\left|\beta\left(\xi-(\alpha / 2)\left(1-\eta^{2}\right)\right)\right| \theta^{q+r}}{|Q| \Gamma(q+r+1)}\right. \\
& +\frac{|\alpha \beta| \theta}{|Q|}\left(\frac{1}{\Gamma(q+r+2)}+\frac{\eta^{q+r+1}}{\Gamma(q+r+2)}\right) \\
& +\frac{|\beta| \theta \xi^{q+r}}{|Q| \Gamma(q+r+1)}+\frac{1}{|Q|} \\
& \quad \times\{|\alpha(1-\beta)| \\
& \quad \times\left(\frac{1}{\Gamma(q+r+2)}+\frac{\eta^{q+r+1}}{\Gamma(q+r+2)}\right) \\
& \left.+\frac{|1-\beta| \xi^{q+r}}{\Gamma(q+r+1)}\right) \\
& \left.\left.+\frac{|\beta(1-\alpha(1-\eta))| \theta^{q+r}}{\Gamma(q+r+1)}\right\}\right]
\end{aligned}
$$

Using $|f(t, x(t))| \leq|f(t, x(t))-f(t, 0)|+|f(t, 0)| \leq L v+$ $M$, for $x \in B_{v}, t \in[0,1]$, we get

$$
\left|\left(\mathscr{P}_{1} x\right)(t)\right|
$$

$$
\leq|A|(L \nu+M)
$$

$$
\begin{gathered}
\times\left\{\frac{1}{\Gamma(q+1)}+\frac{\left|\beta\left(\xi-(\alpha / 2)\left(1-\eta^{2}\right)\right)\right| \theta^{q}}{|Q| \Gamma(q+1)}\right. \\
+\frac{|\alpha \beta| \theta}{|Q|}\left(\frac{1}{\Gamma(q+2)}+\frac{\eta^{q+1}}{\Gamma(q+2)}\right)
\end{gathered}
$$




$$
\begin{aligned}
& +\frac{|\beta| \theta \xi^{q}}{|Q| \Gamma(q+1)}+\frac{1}{|Q|} \\
& \times\left[|\alpha(1-\beta)|\left(\frac{1}{\Gamma(q+2)}+\frac{\eta^{q+1}}{\Gamma(q+2)}\right)\right. \\
& +\frac{|1-\beta| \xi^{q}}{\Gamma(q+1)} \\
& \left.\left.+\frac{|\beta(1-\alpha(1-\eta))| \theta^{q}}{\Gamma(q+1)}\right]\right\}, \\
& \times\left\{\frac{1}{\Gamma(q+r+1)}\right. \\
& +\frac{\left|\beta\left(\xi-(\alpha / 2)\left(1-\eta^{2}\right)\right)\right| \theta^{q+r}}{|Q| \Gamma(q+r+1)} \\
& +\frac{|\alpha \beta| \theta}{|Q|}\left(\frac{1}{\Gamma(q+r+2)}+\frac{\eta^{q+r+1}}{\Gamma(q+r+2)}\right) \\
& +\frac{|\beta| \theta \xi^{q+r}}{|Q| \Gamma(q+r+1)}+\frac{1}{|Q|} \\
& \times\left[|\alpha(1-\beta)|\left(\frac{1}{\Gamma(q+r+2)}+\frac{\eta^{q+r+1}}{\Gamma(q+r+2)}\right)\right. \\
& +\frac{|1-\beta| \xi^{q+r}}{\Gamma(q+r+1)} \\
& \left.\left.+\frac{|\beta(1-\alpha(1-\eta))| \theta^{q+r}}{\Gamma(q+r+1)}\right]\right\},
\end{aligned}
$$

where we have used the following relations:

$$
\begin{aligned}
& \int_{0}^{t} \frac{(t-s)^{q-1}}{\Gamma(q)} \int_{0}^{s} \frac{(s-u)^{r-1}}{\Gamma(r)} d u d s \\
& \quad=\int_{0}^{t} \frac{(t-s)^{q-1}}{\Gamma(q)} \frac{(s)^{r}}{\Gamma(r+1)} d s \\
& \quad=\frac{t^{q+r}}{\Gamma(q) \Gamma(r+1)} \int_{0}^{1}(1-v)^{q-1} v^{r} d v \\
& \quad=\frac{t^{q+r}}{\Gamma(q+r+1)}, \\
& \int_{0}^{1}(1-u)^{\alpha-1} u^{\beta} d u=\frac{\Gamma(\alpha) \Gamma(\beta+1)}{\Gamma(\alpha+\beta+1)} .
\end{aligned}
$$

Consequently

$$
\begin{aligned}
& |(\mathscr{P} x)(t)| \\
& \quad \leq\left|\left(\mathscr{P}_{1} x\right)(t)\right|+\left|\left(\mathscr{P}_{2} x\right)(t)\right|
\end{aligned}
$$

$$
\begin{aligned}
\leq|A| & (L \nu+M) \\
\times\{ & \frac{1}{\Gamma(q+1)}+\frac{\left|\beta\left(\xi-(\alpha / 2)\left(1-\eta^{2}\right)\right)\right| \theta^{q}}{|Q| \Gamma(q+1)} \\
& +\frac{|\alpha \beta| \theta}{|Q|}\left(\frac{1}{\Gamma(q+2)}+\frac{\eta^{q+1}}{\Gamma(q+2)}\right) \\
& +\frac{|\beta| \theta \xi^{q}}{|Q| \Gamma(q+1)}+\frac{1}{|Q|} \\
& \times\left[|\alpha(1-\beta)|\left(\frac{1}{\Gamma(q+2)}+\frac{\eta^{q+1}}{\Gamma(q+2)}\right)\right. \\
+|B| & \left.\left.+\frac{|1-\beta| \psi(\nu)}{\Gamma(q+1)}+\frac{|\beta(1-\alpha(1-\eta))| \theta^{q}}{\Gamma(q+1)}\right]\right\} \\
\times\{ & \frac{1}{\Gamma(q+r+1)}+\frac{\left|\beta\left(\xi-(\alpha / 2)\left(1-\eta^{2}\right)\right)\right| \theta^{q+r}}{|Q| \Gamma(q+r+1)} \\
+\gamma \nu+\omega \leq v, & \frac{|\alpha \beta| \theta}{|Q|}\left(\frac{1}{\Gamma(q+r+2)}+\frac{\eta^{q+r+1}}{\Gamma(q+r+2)}\right) \\
+ & \frac{|\beta| \theta \xi^{q+r+r}}{|Q| \Gamma(q+r+1)}+\frac{1}{|Q|} \\
\times & {\left[|\alpha(1-\beta)|\left(\frac{|1-\beta| \xi^{q+r}}{\Gamma(q+r+2)}+\frac{|\beta(1-\alpha(1-\eta))| \theta^{q+r}}{\Gamma(q+r+2)}\right]\right\} }
\end{aligned}
$$

which implies that $(\mathscr{P} x)\left(B_{\nu}\right) \subset B_{\nu}$.

Step $2\left(\mathscr{P}_{1}\right.$ is continuous and $\gamma$-contractive). To show the continuity of $\mathscr{P}_{1}$ for $t \in[0,1]$, let us consider a sequence $x_{n}$ converging to $x$. Then, by the assumption $\left(H_{1}\right)$, we have

$$
\begin{aligned}
\mid\left(\mathscr{P}_{1} x_{n}\right)(t) & -\left(\mathscr{P}_{1} x\right)(t) \mid \\
\leq|A| L & \left\{\frac{1}{\Gamma(q+1)}+\frac{\left|\beta\left(\xi-(\alpha / 2)\left(1-\eta^{2}\right)\right)\right| \theta^{q}}{|Q| \Gamma(q+1)}\right. \\
& +\frac{|\alpha \beta| \theta}{|Q|}\left(\frac{1}{\Gamma(q+2)}+\frac{\eta^{q+1}}{\Gamma(q+2)}\right) \\
& +\frac{|\beta| \theta \xi^{q}}{|Q| \Gamma(q+1)}+\frac{1}{|Q|}
\end{aligned}
$$




$$
\begin{array}{r}
\times\left[|\alpha(1-\beta)|\left(\frac{1}{\Gamma(q+2)}+\frac{\eta^{q+1}}{\Gamma(q+2)}\right)\right. \\
\left.\left.\quad+\frac{|1-\beta| \xi^{q}}{\Gamma(q+1)}+\frac{|\beta(1-\alpha(1-\eta))| \theta^{q}}{\Gamma(q+1)}\right]\right\}
\end{array}
$$

$\times\left\|x_{n}-x\right\|$.

Next, we show that $\mathscr{P}_{1}$ is $\gamma$-contractive. For $x, y \in B_{\gamma}$, we get

$$
\begin{aligned}
& \mid\left(\mathscr{P}_{1} x\right)(t)-\left(\mathscr{P}_{1} y\right)(t) \mid \\
& \leq|A| L\left\{\frac{1}{\Gamma(q+1)}+\frac{\left|\beta\left(\xi-(\alpha / 2)\left(1-\eta^{2}\right)\right)\right| \theta^{q}}{|Q| \Gamma(q+1)}\right. \\
&+\frac{|\alpha \beta| \theta}{|Q|}\left(\frac{1}{\Gamma(q+2)}+\frac{\eta^{q+1}}{\Gamma(q+2)}\right) \\
&+\frac{|\beta| \theta \xi^{q}}{|Q| \Gamma(q+1)}+\frac{1}{|Q|} \\
& \times\left[|\alpha(1-\beta)|\left(\frac{1}{\Gamma(q+2)}+\frac{\eta^{q+1}}{\Gamma(q+2)}\right)\right. \\
&\left.\left.+\frac{|1-\beta| \xi^{q}}{\Gamma(q+1)}+\frac{|\beta(1-\alpha(1-\eta))| \theta^{q}}{\Gamma(q+1)}\right]\right\} \\
& \times\|x-y\| .
\end{aligned}
$$

By the given assumption

$$
\begin{aligned}
\gamma:=|A| L\{ & \frac{1}{\Gamma(q+1)} \\
& +\frac{\left|\beta\left(\xi-(\alpha / 2)\left(1-\eta^{2}\right)\right)\right| \theta^{q}}{|Q| \Gamma(q+1)} \\
& +\frac{|\alpha \beta| \theta}{|Q|}\left(\frac{1}{\Gamma(q+2)}+\frac{\eta^{q+1}}{\Gamma(q+2)}\right) \\
& +\frac{|\beta| \theta \xi^{q}}{|Q| \Gamma(q+1)}+\frac{1}{|Q|} \\
& \times\left[|\alpha(1-\beta)|\left(\frac{1}{\Gamma(q+2)}+\frac{\eta^{q+1}}{\Gamma(q+2)}\right)\right. \\
& \left.\left.\quad+\frac{|1-\beta| \xi^{q}}{\Gamma(q+1)}+\frac{|\beta(1-\alpha(1-\eta))| \theta^{q}}{\Gamma(q+1)}\right]\right\}<1,
\end{aligned}
$$

it follows that $\mathscr{P}_{1}$ is $\gamma$-contractive.

Step $3\left(\mathscr{P}_{2}\right.$ is compact). In Step 1 , it has been shown that $\mathscr{P}_{2}$ is uniformly bounded. Now we show that $\mathscr{P}_{2}$ maps bounded sets into equicontinuous sets of $C([0,1], \mathbb{R})$. Let $t_{1}, t_{2} \in[0,1]$ with $t_{1}<t_{2}$ and $x \in B_{\gamma}$. Then we obtain

$$
\begin{aligned}
\left|\left(\mathscr{P}_{2} x\right)\left(t_{2}\right)-\left(\mathscr{P}_{2} x\right)\left(t_{1}\right)\right| & \\
\leq & \frac{|B| \psi(v)}{\Gamma(q+r+1)} \\
& \times \int_{0}^{t_{1}}\left[\left(t_{2}-s\right)^{q-1}-\left(t_{1}-s\right)^{q-1}\right] m(s) d s \\
& +\frac{|B| \psi(v)}{\Gamma(q+r+1)} \int_{t_{1}}^{t_{2}}\left(t_{2}-s\right)^{q-1} m(s) d s \\
+ & \frac{|B| \psi(v)\|m\|\left|t_{2}-t_{1}\right|}{|Q|} \\
\quad & {\left[|\alpha(1-\beta)|\left(\frac{1}{\Gamma(q+r+2)}+\frac{\eta^{q+r+1}}{\Gamma(q+r+2)}\right)\right.} \\
& \left.\quad+\frac{|1-\beta| \xi^{q+r}}{\Gamma(q+r+1)}+\frac{|\beta(1-\alpha(1-\eta))| \theta^{q+r}}{\Gamma(q+r+1)}\right] .
\end{aligned}
$$

Obviously the right hand side of the above inequality tends to zero independently of $x \in B_{v}$ as $t_{2}-t_{1} \rightarrow 0$. Therefore it follows by the Arzelá-Ascoli theorem that $\mathscr{P}_{2}: C([0$, $1], \mathbb{R}) \rightarrow C([0,1], \mathbb{R})$ is completely continuous. Thus $\mathscr{P}_{2}$ is compact on $[0,1]$.

Step 4 ( $\mathscr{P}$ is condensing). Since $\mathscr{P}_{1}$ is continuous, $\gamma$-contractive and $\mathscr{P}_{2}$ is compact, by Lemma 6, $\mathscr{P}: B_{v} \rightarrow B_{v}$ with $\mathscr{P}=\mathscr{P}_{1}+\mathscr{P}_{2}$ is a condensing map on $B_{v}$.

Consequently, by Theorem 7, the map $\mathscr{P}$ has a fixed point which, in turn, implies that the problem (1) has a solution.

Example 9. Consider a nonlocal integral boundary value problem of fractional integrodifferential equations given by

$$
\begin{aligned}
D^{3 / 2} x(t) & =f(t, x(t))+I^{3 / 4} g(t, x(t)), \quad t \in[0,1], \\
x(0) & =\frac{1}{2} x\left(\frac{1}{4}\right), \quad x\left(\frac{1}{3}\right)=\int_{2 / 3}^{1} x(s) d s,
\end{aligned}
$$

where $q=3 / 2, A=B=1, r=3 / 4, \theta=1 / 4, \xi=1 / 3, \eta=$ $2 / 3, \alpha=1, \beta=1 / 2, f(t, x)=\left(1 /(2+t)^{3}\right) \tan ^{-1} x+t+1$, and $g(t, x)=\left(t^{2} /\left(1+t^{3}\right)\right)(1+(|x| /(1+|x|)))$.

Clearly $L=1 / 8$ as $|f(t, x)-f(t, y)| \leq(1 / 8)|x-y|$, and $|g(t, x)| \leq m(t) \psi(\|x\|)$ with $m(t)=t^{2} /\left(1+t^{3}\right)$ and $\psi(\|x\|)=2$. Furthermore $|Q|=1 / 9$, and the condition (16) yields $\gamma \simeq 0.522371<1$. Thus all the conditions of Theorem 8 are satisfied and consequently the problem (26) has a solution.

3.2. Existence Results via O'Regan's Fixed Point Theorem. Our next existence result relies on a fixed point theorem due to O'Regan in [21]. 
Lemma 10. Denote by $U$ an open set in a closed, convex set $C$ of a Banach space E. Assume $0 \in U$. Also assume that $F(\bar{U})$ is bounded and that $F: \bar{U} \rightarrow C$ is given by $F=F_{1}+F_{2}$, in which $F_{1}: \bar{U} \rightarrow E$ is continuous and completely continuous and $F_{2}: \bar{U} \rightarrow E$ is a nonlinear contraction (i.e., there exists a nonnegative nondecreasing function $\phi:[0, \infty) \rightarrow[0, \infty)$ satisfying $\phi(z)<z$ for $z>0$, such that $\left\|F_{2}(x)-F_{2}(y)\right\| \leq$ $\phi(\|x-y\|)$ for all $x, y \in \bar{U})$. Then, either

(C1) F has a fixed point $u \in \bar{U}$, or

(C2) there exist a point $u \in \partial U$ and $\lambda \in(0,1)$ with $u=\lambda F(u)$, where $\bar{U}$ and $\partial U$, respectively, represent the closure and boundary of $U$.

For convenience we set

$$
\begin{aligned}
& p_{0}=|A|\left\{\frac{1}{\Gamma(q+1)}+\frac{\left|\beta\left(\xi-(\alpha / 2)\left(1-\eta^{2}\right)\right)\right| \theta^{q}}{|Q| \Gamma(q+1)}\right. \\
& +\frac{|\alpha \beta| \theta}{|Q|}\left(\frac{1}{\Gamma(q+2)}+\frac{\eta^{q+1}}{\Gamma(q+2)}\right) \\
& +\frac{|\beta| \theta \xi^{q}}{|Q| \Gamma(q+1)}+\frac{1}{|Q|} \\
& \times\left[|\alpha(1-\beta)|\left(\frac{1}{\Gamma(q+2)}+\frac{\eta^{q+1}}{\Gamma(q+2)}\right)\right. \\
& \left.\left.+\frac{|1-\beta| \xi^{q}}{\Gamma(q+1)}+\frac{|\beta(1-\alpha(1-\eta))| \theta^{q}}{\Gamma(q+1)}\right]\right\}, \\
& k_{0}=|B|\left\{\frac{1}{\Gamma(q+r+1)}+\frac{\left|\beta\left(\xi-(\alpha / 2)\left(1-\eta^{2}\right)\right)\right| \theta^{q+r}}{|Q| \Gamma(q+r+1)}\right. \\
& +\frac{|\alpha \beta| \theta}{|Q|}\left(\frac{1}{\Gamma(q+r+2)}+\frac{\eta^{q+r+1}}{\Gamma(q+r+2)}\right) \\
& +\frac{|\beta| \theta \xi^{q+r}}{|Q| \Gamma(q+r+1)}+\frac{1}{|Q|} \\
& \times\left[|\alpha(1-\beta)|\left(\frac{1}{\Gamma(q+r+2)}+\frac{\eta^{q+r+1}}{\Gamma(q+r+2)}\right)\right. \\
& \left.\left.+\frac{|1-\beta| \xi^{q+r}}{\Gamma(q+r+1)}+\frac{|\beta(1-\alpha(1-\eta))| \theta^{q+r}}{\Gamma(q+r+1)}\right]\right\} .
\end{aligned}
$$

Let

$$
\Omega_{\sigma}=\{x \in C([0,1], \mathbb{R}):\|x\|<\sigma\}
$$

and denote the maximum number by

$$
M_{\sigma}=\max \{|f(t, x)|:(t, x) \in[0,1] \times[-\sigma, \sigma]\} .
$$

Theorem 11. Let $f, g:[0,1] \times \mathbb{R} \rightarrow \mathbb{R}$ be continuous functions. Assume that

$\left(A_{1}\right)$ there exist a nonnegative function $p \in C([0,1], \mathbb{R})$ and a nondecreasing function $\zeta:[0, \infty) \rightarrow(0, \infty)$ such that

$|f(t, u)| \leq p(t) \zeta(\|u\|) \quad$ for any $(t, u) \in[0,1] \times \mathbb{R}$

$\left(A_{2}\right)$ there exist a positive constant $\ell<k_{0}^{-1}$ and a continuous function $\phi:[0, \infty) \rightarrow(0, \infty)$ such that $\phi(z) \leq \ell z$ and $|g(t, u)-g(t, v)| \leq \phi(\|u-v\|)$ for all $t \in[0,1]$ and $u, v \in \mathbb{R}$;

$\left(A_{3}\right) \sup _{r \in(0, \infty)}\left(r /\left(k_{0} K+p_{0} \zeta(r)\|p\|\right)\right)>\left(1 /\left(1-k_{0} \ell\right)\right)$, where $K=\sup _{t \in[0,1]}|g(t, 0)|$.

Then the boundary value problem (1) has at least one solution on $[0,1]$.

Proof. By the assumption $\left(\mathrm{A}_{3}\right)$, there exists a number $r_{0}>0$ such that

$$
\frac{r_{0}}{k_{0} K+p_{0} \zeta\left(r_{0}\right)\|p\|}>\frac{1}{1-k_{0} \ell} .
$$

We will show that the operators $\mathscr{P}_{1}$ and $\mathscr{P}_{2}$ defined by (12) and (13), respectively, satisfy all the conditions of Lemma 10. The proof consists of a series of steps.

Step 1 (the operator $\mathscr{P}_{1}$ is continuous and completely continuous). We first show that $\mathscr{P}_{1}\left(\bar{\Omega}_{r_{0}}\right)$ is bounded. For any $x \in \bar{\Omega}_{r_{0}}$, we have

$$
\begin{aligned}
& \left\|\left(\mathscr{P}_{1} x\right)\right\| \\
& \leq|A| M_{\sigma} \\
& \quad \times\left\{\frac{1}{\Gamma(q+1)}+\frac{\left|\beta\left(\xi-(\alpha / 2)\left(1-\eta^{2}\right)\right)\right| \theta^{q}}{|Q| \Gamma(q+1)}\right. \\
& +\frac{|\alpha \beta| \theta}{|Q|}\left(\frac{1}{\Gamma(q+2)}+\frac{\eta^{q+1}}{\Gamma(q+2)}\right) \\
& +\frac{|\beta| \theta \xi^{q}}{|Q| \Gamma(q+1)}+\frac{1}{|Q|} \\
& \times\left[|\alpha(1-\beta)|\left(\frac{1}{\Gamma(q+2)}+\frac{\eta^{q+1}}{\Gamma(q+2)}\right)\right.
\end{aligned}
$$

$$
\left.\left.+\frac{|1-\beta| \xi^{q}}{\Gamma(q+1)}+\frac{|\beta(1-\alpha(1-\eta))| \theta^{q}}{\Gamma(q+1)}\right]\right\} .
$$


Thus the operator $\mathscr{P}_{1}\left(\bar{\Omega}_{r_{0}}\right)$ is uniformly bounded. For any $t_{1}, t_{2} \in[0,1], t_{1}<t_{2}$, we have

$$
\begin{aligned}
& \left|\left(\mathscr{P}_{1} x\right)\left(t_{2}\right)-\left(\mathscr{P}_{1} x\right)\left(t_{1}\right)\right| \\
& \leq|A| M_{r}\left[\int_{0}^{t_{1}}\left[\left(t_{2}-s\right)^{q-1}-\left(t_{1}-s\right)^{q-1}\right] d s\right. \\
& \left.\quad+\int_{t_{1}}^{t_{2}}\left(t_{2}-s\right)^{q-1} d s\right] \\
& +\frac{|A| M_{r}\left|t_{2}-t_{1}\right|}{|Q|} \\
& \times\left[|\alpha(1-\beta)|\left(\frac{1}{\Gamma(q+2)}+\frac{\eta^{q+1}}{\Gamma(q+2)}\right)\right. \\
& \left.\quad+\frac{|1-\beta| \xi^{q}}{\Gamma(q+1)}+\frac{|\beta(1-\alpha(1-\eta))| \theta^{q}}{\Gamma(q+1)}\right],
\end{aligned}
$$

which is independent of $x$ and tends to zero as $t_{2}-t_{1} \rightarrow$ 0 . Thus, $\mathscr{P}_{1}$ is equicontinuous. Hence, by the Arzelá-Ascoli theorem, $\mathscr{P}_{1}\left(\bar{\Omega}_{r_{0}}\right)$ is a relatively compact set. Now, let $x_{n} \in$ $\bar{\Omega}_{r_{0}}$ with $\left\|x_{n}-x\right\| \rightarrow 0$. Then the limit $\left\|x_{n}(t)-x(t)\right\| \rightarrow 0$ is uniformly valid on $[0,1]$. From the uniform continuity of $f(t, x)$ on the compact set $[0,1] \times\left[-r_{0}, r_{0}\right]$, it follows that $\left\|f\left(t, x_{n}(t)\right)-f(t, x(t))\right\| \rightarrow 0$ is uniformly valid on $[0,1]$. Hence $\left\|\mathscr{P}_{1} x_{n}-\mathscr{P}_{1} x\right\| \rightarrow 0$ as $n \rightarrow \infty$. This shows the continuity of $\mathscr{P}_{1}$.

Step 2 (the operator $\mathscr{P}_{2}: \bar{\Omega}_{r_{0}} \rightarrow C([0,1], \mathbb{R})$ is contractive). Consider

$$
\begin{aligned}
\mid\left(\mathscr{P}_{2} x\right)(t) & -\left(\mathscr{P}_{2} y\right)(t) \mid \\
\leq|B|\{ & \frac{1}{\Gamma(q+r+1)} \\
& +\frac{\left|\beta\left(\xi-(\alpha / 2)\left(1-\eta^{2}\right)\right)\right| \theta^{q+r}}{|Q| \Gamma(q+r+1)} \\
& +\frac{|\alpha \beta| \theta}{|Q|}\left(\frac{1}{\Gamma(q+r+2)}+\frac{\eta^{q+r+1}}{\Gamma(q+r+2)}\right) \\
& +\frac{|\beta| \theta \xi^{q+r}}{|Q| \Gamma(q+r+1)}+\frac{1}{|Q|} \\
& \times\left[|\alpha(1-\beta)|\left(\frac{1}{\Gamma(q+r+2)}+\frac{\eta^{q+r+1}}{\Gamma(q+r+2)}\right)\right. \\
\times \phi( & \left.\left.+\frac{|1-\beta| \xi^{q+r}}{\Gamma(q+r+1)}+\frac{|\beta(1-\alpha(1-\eta))| \theta^{q+r}}{\Gamma(q+r+1)}\right]\right\}
\end{aligned}
$$

This, together with $\left(\mathrm{A}_{2}\right)$, implies that

$$
\left\|\left(\mathscr{P}_{2} x\right)-\left(\mathscr{P}_{2} y\right)\right\| \leq \phi(\|x-y\|),
$$

so $\mathscr{P}_{2}: \bar{\Omega}_{r_{0}} \rightarrow C([0,1], \mathbb{R})$ is a nonlinear contraction.
Step 3 (the set $\mathscr{P}\left(\bar{\Omega}_{r_{0}}\right)$ is bounded). Using the inequality

$$
\begin{aligned}
|g(t, x)| & \leq|g(t, x)-g(t, 0)|+|g(t, 0)| \\
& \leq \phi(\|x\|)+K \leq \ell r_{0}+K,
\end{aligned}
$$

we have

$$
\begin{aligned}
& \left|\mathscr{P}_{2}(x)\right| \\
& \leq|B|\left(\ell r_{0}+K\right) \\
& \times\left\{\frac{1}{\Gamma(q+r+1)}\right. \\
& +\frac{\left|\beta\left(\xi-(\alpha / 2)\left(1-\eta^{2}\right)\right)\right| \theta^{q+r}}{|Q| \Gamma(q+r+1)} \\
& +\frac{|\alpha \beta| \theta}{|Q|}\left(\frac{1}{\Gamma(q+r+2)}+\frac{\eta^{q+r+1}}{\Gamma(q+r+2)}\right) \\
& +\frac{|\beta| \theta \xi^{q+r}}{|Q| \Gamma(q+r+1)}+\frac{1}{|Q|} \\
& \times\left[|\alpha(1-\beta)|\left(\frac{1}{\Gamma(q+r+2)}+\frac{\eta^{q+r+1}}{\Gamma(q+r+2)}\right)\right. \\
& \left.\left.+\frac{|1-\beta| \xi^{q+r}}{\Gamma(q+r+1)}+\frac{|\beta(1-\alpha(1-\eta))| \theta^{q+r}}{\Gamma(q+r+1)}\right]\right\},
\end{aligned}
$$

for any $x \in \bar{\Omega}_{r_{0}}$. This, with the boundedness of the set $\mathscr{P}_{1}\left(\bar{\Omega}_{r_{0}}\right)$, implies that the set $\mathscr{P}\left(\bar{\Omega}_{r_{0}}\right)$ is bounded.

Step 4 (finally, it will be shown that the case (C2) in Lemma 10 does not hold). On the contrary, we suppose that (C2) holds. Then, we have that there exist $\lambda \in(0,1)$ and $x \in \partial \Omega_{r_{0}}$ such that $x=\lambda \mathscr{P} x$. So, we have $\|x\|=r_{0}$ and

$$
\begin{aligned}
x(t)= & \lambda A \int_{0}^{t} \frac{(t-s)^{q-1}}{\Gamma(q)} f(s, x(s)) d s \\
& +\lambda A \frac{\beta\left(\xi-(\alpha / 2)\left(1-\eta^{2}\right)\right)}{Q} \\
& \times \int_{0}^{\theta} \frac{(\theta-s)^{q-1}}{\Gamma(q)} f(s, x(s)) d s \\
& +\lambda A \frac{\alpha \beta \theta}{Q}\left(\int_{0}^{1} \frac{(1-s)^{q}}{\Gamma(q+1)} f(s, x(s)) d s\right. \\
& -\lambda A \frac{\beta \theta}{Q} \int_{0}^{\xi} \frac{(\xi-s)^{q}}{\Gamma(q)} f(s, x(s)) d s+\frac{\lambda A t}{Q}
\end{aligned}
$$




$$
\begin{aligned}
& \times\left[\alpha ( 1 - \beta ) \left(\int_{0}^{1} \frac{(1-s)^{q}}{\Gamma(q+1)} f(s, x(s)) d s\right.\right. \\
& \left.-\int_{0}^{\eta} \frac{(\eta-s)^{q}}{\Gamma(q+1)} f(s, x(s)) d s\right) \\
& -(1-\beta) \int_{0}^{\xi} \frac{(\xi-s)^{q}}{\Gamma(q)} f(s, x(s)) d s \\
& -\beta(1-\alpha(1-\eta)) \int_{0}^{\theta} \frac{(\theta-s)^{q-1}}{\Gamma(q)} \\
& \times f(s, x(s)) d s] \\
& +\lambda B \int_{0}^{t} \frac{(t-s)^{q-1}}{\Gamma(q)} \int_{0}^{s} \frac{(s-u)^{r-1}}{\Gamma(r)} g(u, x(u)) d u d s \\
& +\lambda B \frac{\beta\left(\xi-(\alpha / 2)\left(1-\eta^{2}\right)\right)}{Q} \\
& \times \int_{0}^{\theta} \frac{(\theta-s)^{q-1}}{\Gamma(q)} \int_{0}^{s} \frac{(s-u)^{r-1}}{\Gamma(r)} g(u, x(u)) d u d s \\
& +\lambda B \frac{\alpha \beta \theta}{Q}\left(\int_{0}^{1} \frac{(1-s)^{q}}{\Gamma(q+1)} \int_{0}^{s} \frac{(s-u)^{r-1}}{\Gamma(r)}\right. \\
& \times g(u, x(u)) d u d s \\
& -\int_{0}^{\eta} \frac{(\eta-s)^{q}}{\Gamma(q+1)} \int_{0}^{s} \frac{(s-u)^{r-1}}{\Gamma(r)} \\
& \times g(u, x(u)) d u d s) \\
& -\lambda B \frac{\beta \theta}{Q} \int_{0}^{\xi} \frac{(\xi-s)^{q}}{\Gamma(q)} \int_{0}^{s} \frac{(s-u)^{r-1}}{\Gamma(r)} g(u, x(u)) d u d s \\
& +\frac{\lambda B t}{Q}[\alpha(1-\beta) \\
& \times\left(\int_{0}^{1} \frac{(1-s)^{q}}{\Gamma(q+1)} \int_{0}^{s} \frac{(s-u)^{r-1}}{\Gamma(r)}\right. \\
& \times g(u, x(u)) d u d s \\
& -\int_{0}^{\eta} \frac{(\eta-s)^{q}}{\Gamma(q+1)} \int_{0}^{s} \frac{(s-u)^{r-1}}{\Gamma(r)} \\
& \times g(u, x(u)) d u d s) \\
& -(1-\beta) \int_{0}^{\xi} \frac{(\xi-s)^{q}}{\Gamma(q)} \int_{0}^{s} \frac{(s-u)^{r-1}}{\Gamma(r)} \\
& \times g(u, x(u)) d u d s \\
& -\beta(1-\alpha(1-\eta))
\end{aligned}
$$

$$
\begin{aligned}
\times \int_{0}^{\theta} \frac{(\theta-s)^{q-1}}{\Gamma(q)} \int_{0}^{s} \frac{(s-u)^{r-1}}{\Gamma(r)} . \\
\times g(u, x(u)) d u d s] .
\end{aligned}
$$

Using the assumptions $\left(A_{1}\right)$ and $\left(A_{2}\right)$, we get

$$
r_{0} \leq k_{0} K+p_{0} \zeta\left(r_{0}\right)\|p\|+k_{0} \ell r_{0},
$$

which leads to a contradiction:

$$
\frac{r_{0}}{k_{0} K+p_{0} \zeta\left(r_{0}\right)\|p\|} \leq \frac{1}{1-k_{0} \ell} .
$$

Thus the operators $\mathscr{P}_{1}$ and $\mathscr{P}_{2}$ satisfy all the conditions of Lemma 10 . Hence, the operator $\mathscr{P}$ has at least one fixed point $x \in \bar{\Omega}_{r_{0}}$, which is the solution of the problem (1). This completes the proof.

Example 12. Consider a nonlocal integral boundary value problem of fractional integrodifferential equations given by

$$
\begin{aligned}
D^{3 / 2} x(t) & =f(t, x(t))+I^{3 / 4} g(t, x(t)), \quad t \in[0,1], \\
x(0) & =\frac{1}{2} x\left(\frac{1}{4}\right), \quad x\left(\frac{1}{3}\right)=\int_{2 / 3}^{1} x(s) d s,
\end{aligned}
$$

where $q=3 / 2, A=B=1, r=3 / 4, \theta=1 / 4, \xi=1 / 3, \eta=$ $2 / 3, \alpha=1, \beta=1 / 2, f(t, x)=(1 / 27)(2 \sqrt{(1+t)}-1) \sin x$, and $g(t, x)=\left(1 /(2+t)^{3}\right)(3+(|x| /(1+|x|)))$.

Observe that $|f(t, x)| \leq(1 / 27)(2 \sqrt{(1+t)}-1) x, \mid g(t, x)-$ $g(t, y)|\leq(1 / 8)| x-y \mid$, and $\sup _{t \in[0,1]} g(t, 0)=3 / 8$. Further, we set $p(t)=(1 / 3)(2 \sqrt{(1+t)}-1), \zeta(x)=x / 9, \ell=1 / 8$, and $K=$ $3 / 8$. With the given data, it is found that $k_{0} \simeq 1.495606, p_{0} \simeq$ 4.178968, $|Q|=1 / 9,\|p\|=(2 \sqrt{2}-1) / 3$, and

$$
\begin{gathered}
\sup _{r \in(0, \infty)}\left\{\frac{r}{k_{0} K+p_{0} \zeta(r)\|p\|}\right\} \simeq 3.533597 \\
\frac{1}{1-k_{0} \ell} \simeq 1.229953 .
\end{gathered}
$$

Clearly, all the conditions of Theorem 11 are satisfied and hence there exists a solution for the problem (41).

\section{Conflict of Interests}

The authors declare that there is no conflict of interests regarding the publication of this paper.

\section{Acknowledgment}

This article was funded by Deanship of Scientific Research (DSR), King Abdulaziz University, Jeddah, Saudi Arabia. The authors, therefore, acknowledge technical and financial support of KAU. 


\section{References}

[1] G. S. Wang and A. F. Blom, "A strip model for fatigue crack growth predictions under general load conditions," Engineering Fracture Mechanics, vol. 40, no. 3, pp. 507-533, 1991.

[2] B. Ahmad, T. Hayat, and S. Asghar, "Diffraction of a plane wave by an elastic knife-edge adjacent to a strip," The Canadian Applied Mathematics Quarterly, vol. 9, pp. 303-316, 2001.

[3] I. Podlubny, Fractional Differential Equations, Academic Press, San Diego, Calif, USA, 1999.

[4] A. A. Kilbas, H. M. Srivastava, and J. J. Trujillo, Theory and Applications of Fractional Differential Equations, NorthHolland Mathematics Studies, Elsevier Science, Amsterdam, The Netherlands, 2006.

[5] J. Sabatier, O. P. Agrawal, and J. A. T. Machado, Eds., Advances in Fractional Calculus: Theoretical Developments and Applications in Physics and Engineering, Springer, Dordrecht, The Netherlands, 2007.

[6] D. Baleanu, K. Diethelm, E. Scalas, and J. J. Trujillo, Fractional Calculus Models and Numerical Methods, Series on Complexity, Nonlinearity and Chaos, World Scientific, Boston, Mass, USA, 2012.

[7] M. Benchohra, S. Hamani, and S. K. Ntouyas, "Boundary value problems for differential equations with fractional order and nonlocal conditions," Nonlinear Analysis: Theory, Methods \& Applications, vol. 71, no. 7-8, pp. 2391-2396, 2009.

[8] W. Zhong and W. Lin, "Nonlocal and multiple-point boundary value problem for fractional differential equations," Computers \& Mathematics with Applications, vol. 59, no. 3, pp. 1345-1351, 2010.

[9] B. Ahmad and S. Sivasundaram, "On four-point nonlocal boundary value problems of nonlinear integro-differential equations of fractional order," Applied Mathematics and Computation, vol. 217, no. 2, pp. 480-487, 2010.

[10] B. Ahmad, "On nonlocal boundary value problems for nonlinear integro-differential equations of arbitrary fractional order," Results in Mathematics, vol. 63, no. 1-2, pp. 183-194, 2013.

[11] B. Ahmad and J. J. Nieto, "Sequential fractional differential equations with three-point boundary conditions," Computers \& Mathematics with Applications, vol. 64, no. 10, pp. 3046-3052, 2012.

[12] J. R. Graef, L. Kong, and Q. Kong, "Application of the mixed monotone operator method to fractional boundary value problems," Fractional Differential Calculus, vol. 2, no. 1, pp. 87-98, 2012.

[13] C. Hu, B. Liu, and S. Xie, "Monotone iterative solutions for nonlinear boundary value problems of fractional differential equation with deviating arguments," Applied Mathematics and Computation, vol. 222, pp. 72-81, 2013.

[14] S. Staněk, "Limit properties of positive solutions of fractional boundary value problems," Applied Mathematics and Computation, vol. 219, no. 5, pp. 2361-2370, 2012.

[15] B. Ahmad and S. K. Ntouyas, "Existence results for nonlocal boundary value problems of fractional differential equations and inclusions with strip conditions," Boundary Value Problems, vol. 2012, 2012.

[16] P. Zhang and Y. Gong, "Existence and multiplicity results for a class of fractional differential inclusions with boundary conditions," Boundary Value Problems, vol. 2012, article 82, 2012.

[17] A. Alsaedi, S. K. Ntouyas, and B. Ahmad, "Existence results for Langevin fractional differential inclusions involving two fractional orders with four-point multiterm fractional integral boundary conditions," Abstract and Applied Analysis, vol. 2013, Article ID 869837, 17 pages, 2013.

[18] R. Kamocki and C. Obczynski, "On fractional differential inclusions with the Jumarie derivative," Journal of Mathematical Physics, vol. 55, no. 2, Article ID 2902, 10 pages, 2014.

[19] J. Tariboon, T. Sitthiwirattham, and S. K. Ntouyas, "Existence results for fractional differential inclusions with multi-point and fractional integral boundary conditions," Journal of Computational Analysis and Applications, vol. 17, no. 2, pp. 343-360, 2014.

[20] B. Ahmad and S. K. Ntouyas, "An existence theorem for fractional hybrid differential inclusions of Hadamard type with Dirichlet boundary conditions," Abstract and Applied Analysis, vol. 2014, Article ID 705809, 7 pages, 2014.

[21] D. O'Regan, "Fixed-point theory for the sum of two operators," Applied Mathematics Letters, vol. 9, no. 1, pp. 1-8, 1996.

[22] A. Granas and J. Dugundji, Fixed Point Theory, Springer, New York, NY, USA, 2005.

[23] E. Zeidler, Nonlinear Functional Analysis and Its Application: Fixed Point-Theorems, vol. 1, Springer, New York, NY, USA, 1986.

[24] B. N. Sadovskii, "On a fixed point principle," Functional Analysis and Its Applications, vol. 1, pp. 74-76, 1967. 


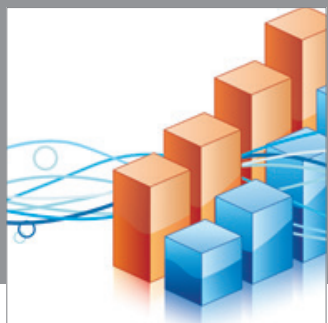

Advances in

Operations Research

mansans

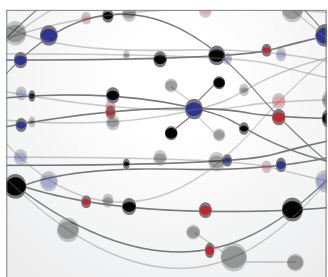

The Scientific World Journal
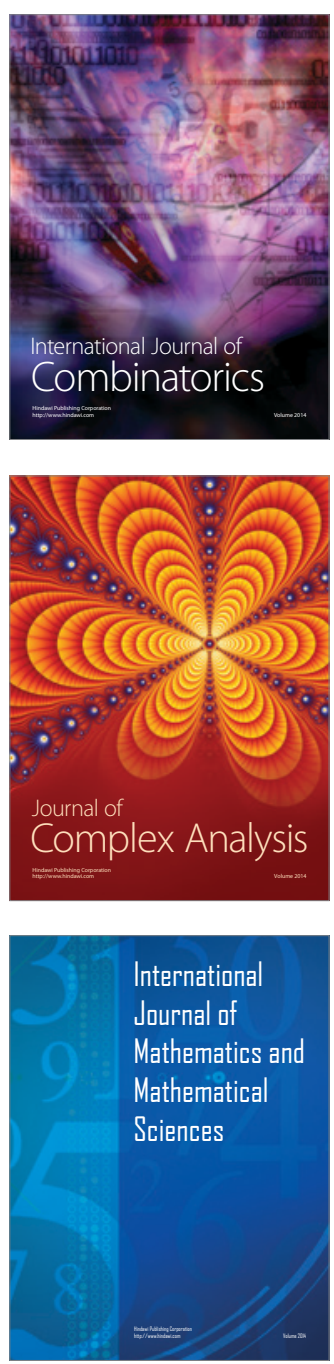
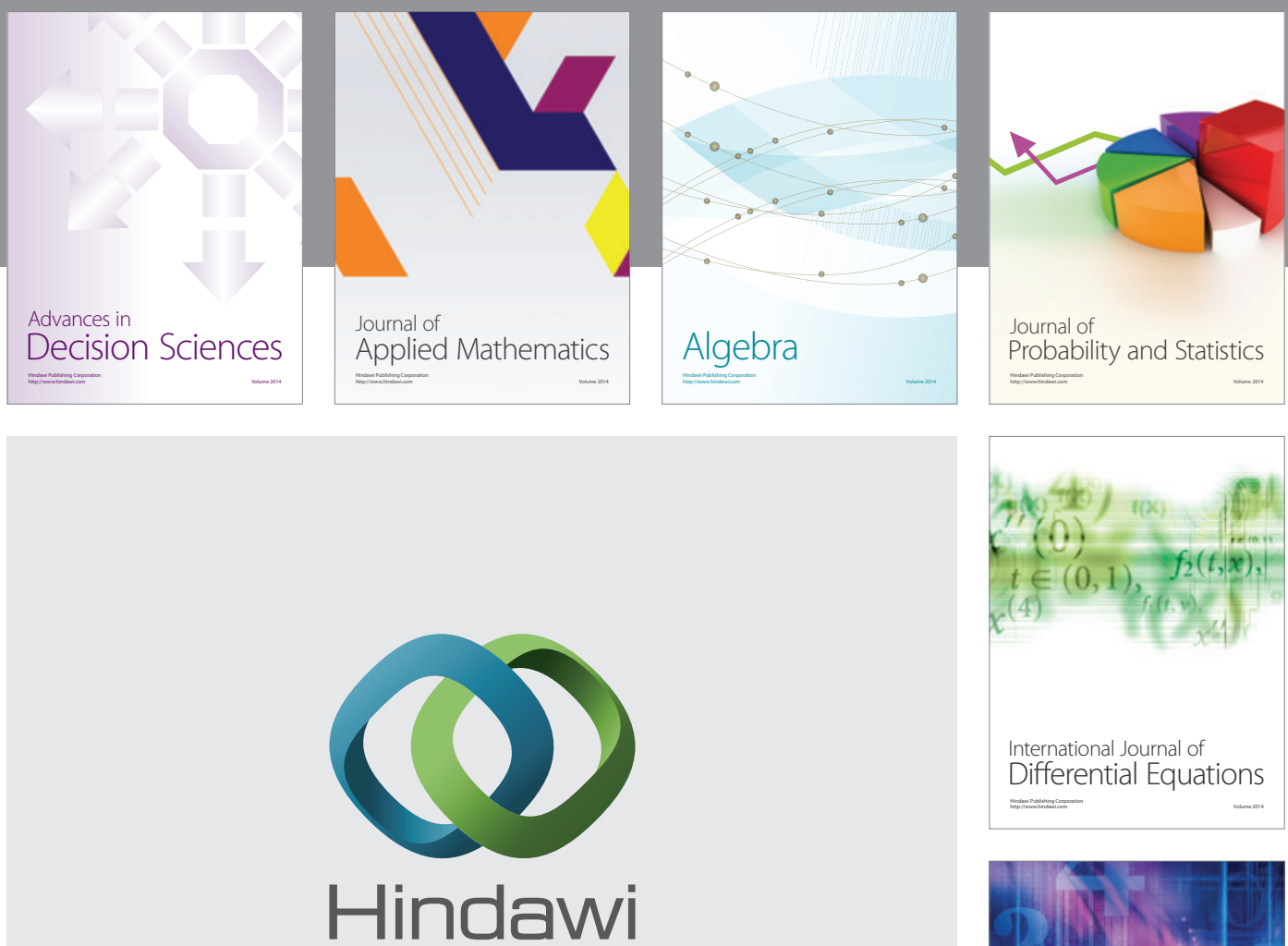

Submit your manuscripts at http://www.hindawi.com
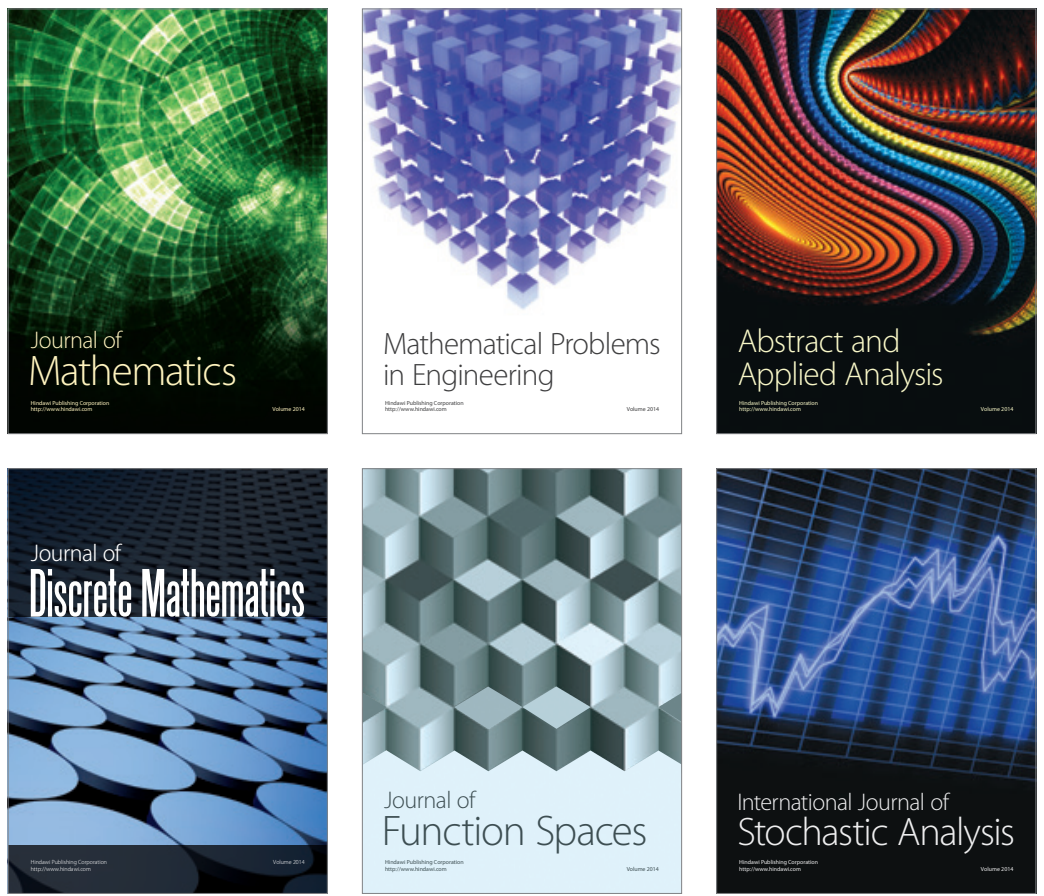

Journal of

Function Spaces

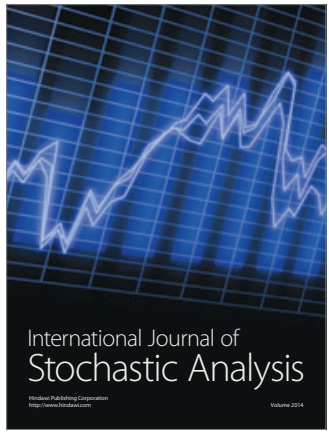

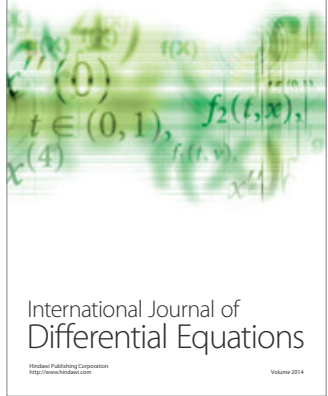
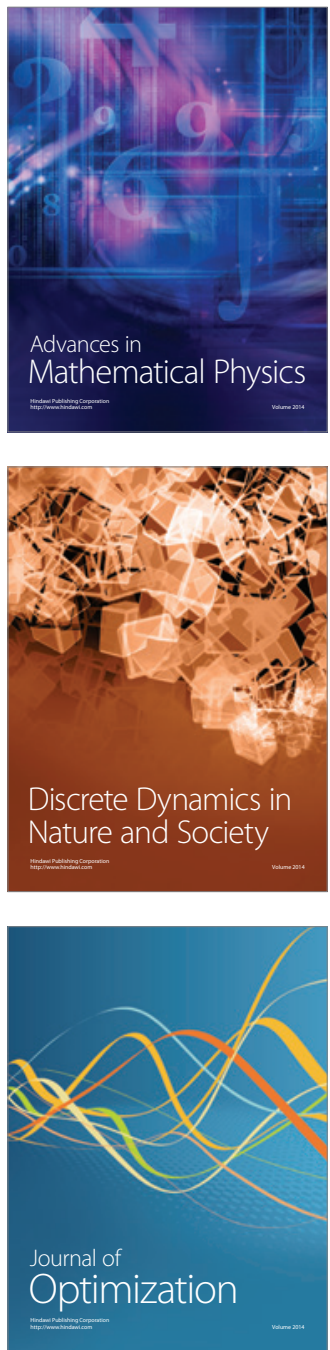